\title{
Habitual sleep duration and the incidence of headaches in college students
}

\author{
ROBERT A. HICKS and JEAN KILCOURSE \\ San Jose State University, San Jose, California 95192
}

\begin{abstract}
The relationship between habitual sleep duration and the frequency of headaches was measured using the responses of 176 students. As was predicted, we found a significant inverse relationship between these variables.
\end{abstract}

As the literature on the relationships between habitual sleep duration and behavior has developed, a pattern of behaviors associated with habitual short sleep (i.e., $\leqslant 6 \mathrm{~h}$ ) has crystallized (e.g., Hartmann, 1973; Hicks, in press). In part, the behaviors associated with this "shortenedsleep syndrome" suggest increased levels of drive, and aggressiveness accompanied by reduced ability to cope with stress.

In summarizing the behaviors of persons who suffer from migraine headaches, Wolff (1963) noted that these people tended to be tense, hard-driving individuals who exhibited difficulty in coping with stress. In taking note of the similarities between these descriptions of behavior, we speculated that there might be an inverse relationship between habitual sleep duration and the frequency of tension or migraine headaches.

\section{METHOD}

To test the hypothesis that there is an inverse relationship between habitual sleep duration and the frequency of headaches, we asked 176 university students to respond to a health-habits questionnaire that included these critical questions: "Estimate the number of hours of sleep that you usually get each night" and "Do you have tension or migraine headaches often? sometimes? never ."

\section{RESULTS AND DISCUSSION}

On the basis of their respones to these questions, each

Please address requests for reprints to Robert A. Hicks, Department of Psychology, San Jose State University, San Jose, California 95192. In part, this research was supported by NIH Grant RR 08192-03 and NIMH Grant MH 17057-01.
Table 1

Frequency in Each Sleep Duration by Headache Frequency Cell

\begin{tabular}{cccc}
\hline Hours of Sleep & Never & Sometimes & Often \\
\hline$\geqslant 8$ & 38 & 17 & 3 \\
7 & 37 & 21 & 5 \\
$\leqslant 6$ & 19 & 31 & 5 \\
\hline
\end{tabular}

student was assigned to one of the nine sleep duration by headache frequency cells that are described in Table 1 . The differences in the distribution of headache frequency between the three sleep duration groupings were tested and found to be significant $\left[\chi^{2}(4)=12.35\right.$, $\mathrm{p}<.02$, with $\left.\varphi^{\prime}=.19\right]$.

Further, the pattern of these distributions is consistent with the hypothesis. That is $66 \%$ of $\geqslant 8$-h sleepers reported never having headaches, $59 \%$ of the 7 -h sleepers reported never having headaches, but only $35 \%$ of the $\leqslant 6$-h sleepers were headache free. Thus, these data indicate that there is a "dose-related" inverse relationship between habitual sleep duration and headaches. In addition, these data suggest to us that there may be other health-related problems that seem to accompany habitually shortened sleep.

\section{REFERENCES}

Hartmann, E. L. The functions of sleep. New Haven, Conn: Yale University Press, 1973.

Hicks, R. A. Helping Type A patients to sleep better. Medical Aspects of Human Sexuality, in press.

WouFr, H. G. Headache and other head pains. New York: Oxford University Press, 1963.

(Received for publication February 4, 1983.) 